\title{
Antibody Distribution on Bio-Functionalized Magnetic Nanoparticles Analyzed by Spatial-Resolved EELS
}

R. Arenal, L. De Matteis, L. Custardoy, A. Mayoral, V. Grazu, J. M. de la Fuente, C. Marquina,M. R. Ibarra

Bio-functionalized nanoparticles have a huge interest due to their use in biotechnology and bio-nanomedicine. However, in order to achieve the functionalization in a more efficient way, a deep knowledge of the bio-functionalizing moieties and their spatial distribution on the nanoparticle surface is required. We have showed that cryo-spatialresolved EELS (SR-EELS) is a very appropriate and powerful technique for providing very rich information at the sub-nanometer scale on complex hybrid nanomaterials. The nanostructures on which we focus in these works are magnetic nanoparticles functionalized with a Protein-G/antibody (PG-Ab) system. SR-EEL spectra were recorded using a VG-HB501 dedicated STEM, operated at $100 \mathrm{kV}$ with an energy resolution close to $0.7-0.8 \mathrm{eV}$ in the core-loss region. Furthermore, in order to avoid electron beam damage, these measurements have been performed using a liquidnitrogen-cooled cryo-stage installed in this microscope, and relatively low electron doses. EELS-STEM studies have been also carried out using a FEI Titan Low-Base microscope, working at $80 \mathrm{kV}$, which is equipped with a Cs probe corrector and ultrabright XFEG electron source.

Figure 1 displays an EEL spectrum-image recorded on PG/Ab-functionalized nanoparticles, where Figure 1 (a) and (b) correspond to the bright-field and high angular annular dark field (HAADF) images of the nanoparticles, respectively. Carbon and nitrogen maps, extracted, after background subtraction, from the EEL spectra displayed in Figure 1 (e), are shown in Figure 1 (c) and (d), respectively. From these maps, we can observe that there is a clear correspondence between the spatial distributions of these elements which are localized at the surface of the nanoparticles. This finding indicates that they correspond to the organic constituents (PG/Ab) biofunctionalizing the magnetic nanoparticles. In this contribution we will also show the analysis of the electron energy loss near edge structure (ELNES) which provides rich information about those materials. In summary, we have showed that the functional moieties (i.e., the antibodies) are only anchored in specific areas of the surface of the nanoparticles. This result showing that the biological entities are discontinuously distributed over the nanoparticle shell is very relevant because validates our selective functionalization protocol. In fact, the use of a critical amount of amino groups on the nanoparticle shell could be tuned for achieving simultaneously good water particle stability and the proper protein $\mathrm{G}$ adsorption for the suitable antibody immobilization on the particle surface leading to a highly efficient immunorecognition. This will have a significant impact on biotechnological applications, as for instance biosensors, where an adequate nanoparticle functionalization approach for antibody immobilization is critical to improve the test sensitivity. 
Figure 1. (a) BF image of an agglomerate of bio-functionalized core-shell-shell nanoparticles. (b) HAADF image of this agglomerate where a 300x300 EELS-SPIM has been recorded at $150 \mathrm{~K}$. (c) and (d) $\mathrm{C}$ and $\mathrm{N}$ chemical maps extracted, after background subtraction, from the EELS SPIM. For the sake of clarity these elemental maps have been colored with a temperature color scale. The individual EELS spectra, after the background removal, corresponding to the sum of the spectra collected in the positions marked in Fig. 1 (c).

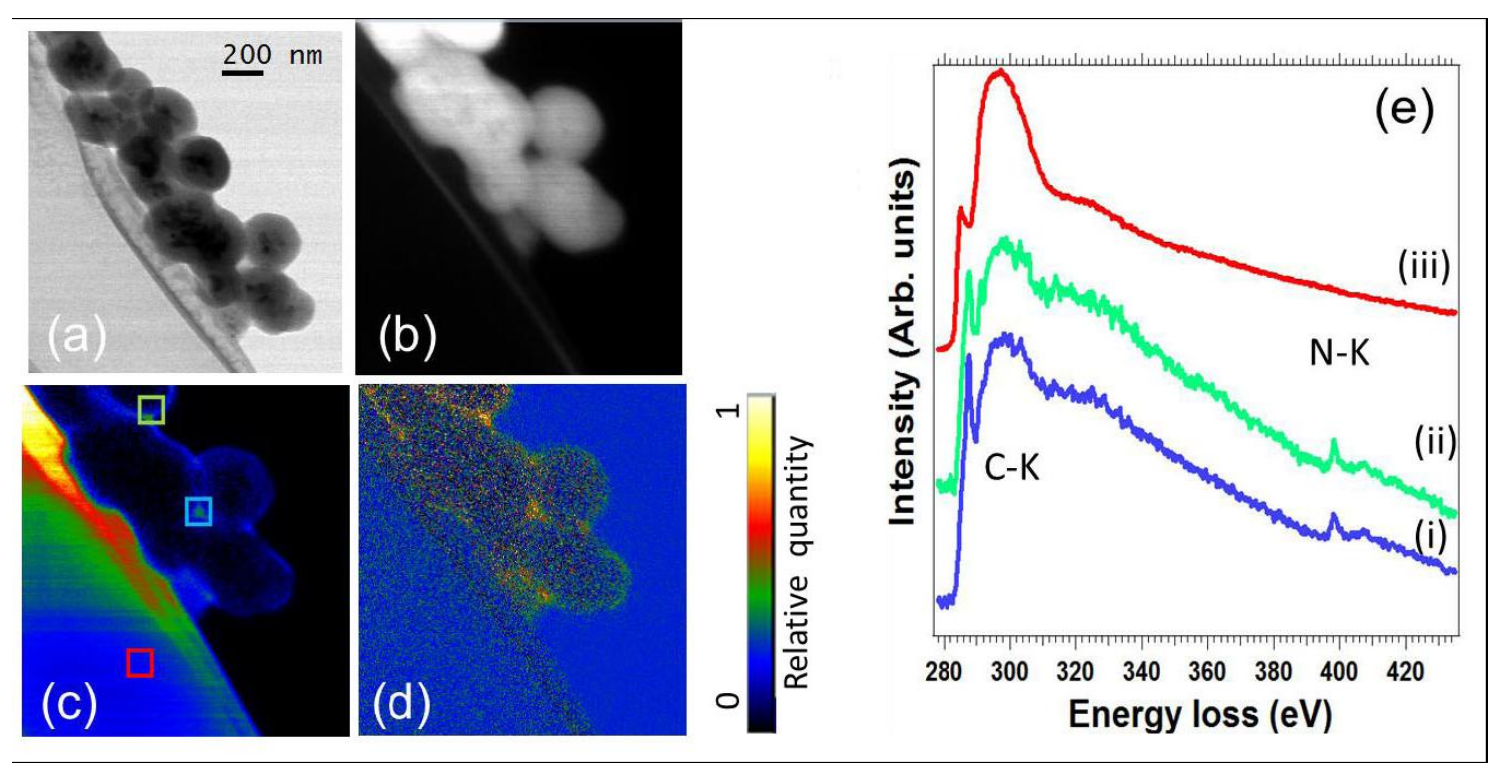

